\title{
Correction to: Marker-assisted identification of novel genetic lines for salinity tolerance and their categorization for utilization in development of hybrid rice (Oryza sativa L.)
}

\author{
P. Raghavendra ${ }^{1}$ - B. M Dushyantha Kumar ${ }^{1}$ P. Shashikumara² ${ }^{2}$. H. M Sachin Kumar ${ }^{1}$ - S. L. Krishnamurthy ${ }^{3}$. \\ Shailaja Hittalmani ${ }^{4}$
}

Published online: 26 November 2020

(c) Akadémiai Kiadó Zrt. 2020

\section{Correction to: Cereal Research Communications https://doi.org/10.1007/s42976-020-00104-2}

Two incorrect marker names were specified in the Abstract of the initial online publication. The original article has been corrected.

https://doi.org/10.1007/s42976-020-00104-2

P. Shashikumara

shashikumarpgpb@gmail.com

P. Raghavendra

raghavendra9078@gmail.com

B. M Dushyantha Kumar

dushyanth123@yahoo.com

H. M Sachin Kumar

hugudisachin@gmail.com

S. L. Krishnamurthy

krishnagene@gmail.com

Shailaja Hittalmani

shailajah_maslab@rediffmail.com

1 Department of Genetics and Plant Breeding, UAHS,

Shivamogga 577204, India

2 ICAR-Indian Grassland and Fodder Research Institute, Jhansi 284003, India

3 ICAR-Central Soil Salinity Research Institute, Karnal, India

4 Department of Genetics and Plant Breeding, UAS, Banglore 560065, India 